\title{
Star formation and chemical evolution of DLAs with semi-analytical models
}

\author{
J. L. Hou ${ }^{1}$, C. G. Shu ${ }^{2,1}$, S. Y. Shen ${ }^{1}$, and R. X. Chang ${ }^{1}$ \\ ${ }^{1}$ Shanghai Astronomical Observatory, CAS, 80 Nandan Road, Shanghai 200030, China \\ ${ }^{2}$ Joint Centre for Astrophysics, Shanghai Normal University, Shanghai, 200234, China \\ email: hjlyx@shao.ac.cn
}

\begin{abstract}
We have examined some basic properties of damped Ly $\alpha$ systems (DLAs) by semianalytic modelling. We assume that DLA hosts are disk galaxies whose mass function is generated by a Press-Schechter formalism at redshift 3. The disk undergoes star formation and chemical evolution. We select modelled DLAs according to their observational criterion by Monte Carlo simulation using random lines of sight and disk inclinations. The DLA ages are set to be 1 to 3 Gyr. By best-fitting the predicted metallicity distribution to the observed ones, we get the effective yield for DLAs of about $0.25 Z_{\odot}$. On the basis of this constraint, we further compared our model predictions with observations at redshift 3 in the following properties: number density, gas content, HI frequency distribution, star formation rate density, and the relationship between metallicity and HI column density. We found that the predicted number density at redshift 3 agrees well with the observed value, but the gas content $\Omega_{D L A}$ is about 3 times larger than observed since our model predicts more DLA systems with higher column density. The frequency distribution at higher HI column density is quite consistent with observations while some difference exists at the lower $\mathrm{HI}$ end. The predicted star formation rate density contributed by DLAs is consistent with the most recent observations. In addition, we have investigated the connection between DLAs and Lyman Break galaxies (LBGs) by comparing their UV luminosity functions, which shows that DLAs host galaxies are much fainter than LBGs. However, there is a discrepancy between the model predictions and observations in the correlation between metallicity and HI column density for DLAs. Further investigations are needed into the mode of star formation in high redshift environments.
\end{abstract}

\section{Introduction}

Quasar absorption line systems are one of the best objects to trace the physical nature of the evolving Universe. Among various absorption systems, damped Ly $\alpha$ systems (DLAs) are the intercepting objects that have highest HI column density. It is often said that DLAs are the progenitors of present-day galaxies. But substantial debate continues over exactly which populations of galaxies are responsible for them. Thanks to the large observing facilities brought on-line during the past decade, more and more data about DLAs are being provided by various observers (see Prochaska et al. 2003).

In order to have a real understanding of the physical nature of DLAs, theorists have used various methods to explain the observed properties, such as simple galaxy models (Hou, Boissier \& Prantzos 2001; Calura, Matteucci \& Vladilo 2003; Boissier, Péroux \& Pettini 2003; Lanfranchi \& Friaca 2003), semi-analytical models (SAMs, Cen et al. 2003; Cora et al. 2003; Hou et al. 2005; Okoshi \& Nagashima 2005), and numerical simulations (Gardner et al. 2001; Nagamine, Springel \& Hernquist 2003; Churches et al. 2004).

In this paper, we will adopt a SAM to examine in detail the observed metallicity, HI column density, and star formation properties of DLAs in the context of the standard hierarchical picture of structure formation in the Universe. The disk galaxy formation 
model with single disks was adopted because we mainly concentrate on HI column densities and the cosmic star formation rate density contribution from DLAs, rather than their kinematics. As an illustration, DLA properties are assumed at redshift $z=3$ and the standard $\Lambda$ CDM cosmogony is adopted.

\section{Model}

\subsection{Galaxy formation}

The galaxy formation model in this paper comes from that for disk galaxies suggested by Mo, Mao \& White (1998, hereafter MMW). In the model of MMW, the halo mass function at any redshift $z$ can be described by the Press-Schechter formalism (PS, Press \& Schechter 1974). The relation between halo mass $M$ and its circular velocity $V_{C}$ is given by $M=V_{C}^{3} /(10 G H(z))$, where $G$ is the gravitational constant, and $H(z)$ is the Hubble constant at redshift $z$. Further details about the model can be found in MMW.

The distribution function of halo spin parameters can be described by a log-normal function based on numerical simulation. Disks are assumed to have exponential surface profiles $\Sigma(R)=\Sigma_{0} \exp \left(-R / R_{d}\right)$, where $\Sigma_{0}$ and $R_{d}$ are the central surface density and the scale length. The disk global properties can be uniquely determined by parameters $\lambda$, $V_{C}, m_{d}$, and the adopted cosmogony, where $\lambda$ is the halo dimensionless spin parameter, $V_{C}$ is the halo circular velocity, and $m_{d}$ is the mass ratio of disk to halo. Here, we have adopted $m_{d}$ as a function of $V_{C}$ in order to consider galactic winds and outflow (Shu et al. 2003).

After knowing the distributions of $V_{C}$ and $\lambda$ for halos, we are able to generate a sample of disk galaxies by Monte-Carlo simulation in the $V_{C}-\lambda$ plane at redshift $z \sim 3$, which is the parent sample for our follow-up DLA simulation.

\subsection{Star formation, chemical evolution, and DLA modelling}

The adopted star formation prescription comes from disk galaxy modelling of Boissier \& Prantzos (2000). Under the approximation of instantaneous recycling, chemical evolution in disks can be expressed by the simple closed-box model with metallicity $Z$ given by $Z-Z_{i}=-p \ln \mu$, where $Z_{i}$ is the initial metallicity of gas and is assumed to be $0.01 Z_{\odot}, p$ is the effective yield, and $\mu$ is the gas fraction. We assume that at the initial time $(t=0$, $z=3)$ the gas surface density $\Sigma_{g 0}(R)=\Sigma_{0} \exp \left(-R / R_{d}\right)$. Star formation proceeds within disks on a typical time-scale of 1 3 Gyr (Lanfranchi \& Friaca 2003; Dessauges-Zavadsky et al. 2004). The effective yield $p$ is assumed to be constant and is obtained by comparing the metallicity distributions between model predictions and observations for DLAs. The modelled DLAs are selected over the sampled galaxies by random sight-lines penetrating disks according to the observed selection criterion, i.e. $N_{\mathrm{HI}} \geqslant 10^{20.3} \mathrm{~cm}^{-2}$. Here, random inclinations for disks in the sky are considered.

\section{Model results and comparison with observations}

\subsection{Observations}

DLAs have many observational properties, including metallicities, column densities, kinematics, etc. The observed metallicities of DLAs adopted in the present paper mainly come from Hou, Boissier \& Prantzos (2001) and Kulkarni \& Fall (2002) for the Zn element. All the data presented by those authors were compiled from the results of various observers. The observed data of HI column densities come from the survey of Storrie-Lombardi \& Wolfe (2000) (hereafter SW00). Since our model focuses on DLAs at redshift $z \sim 3$, the observed properties of DLAs with redshift $z>2$ were selected for comparison. 

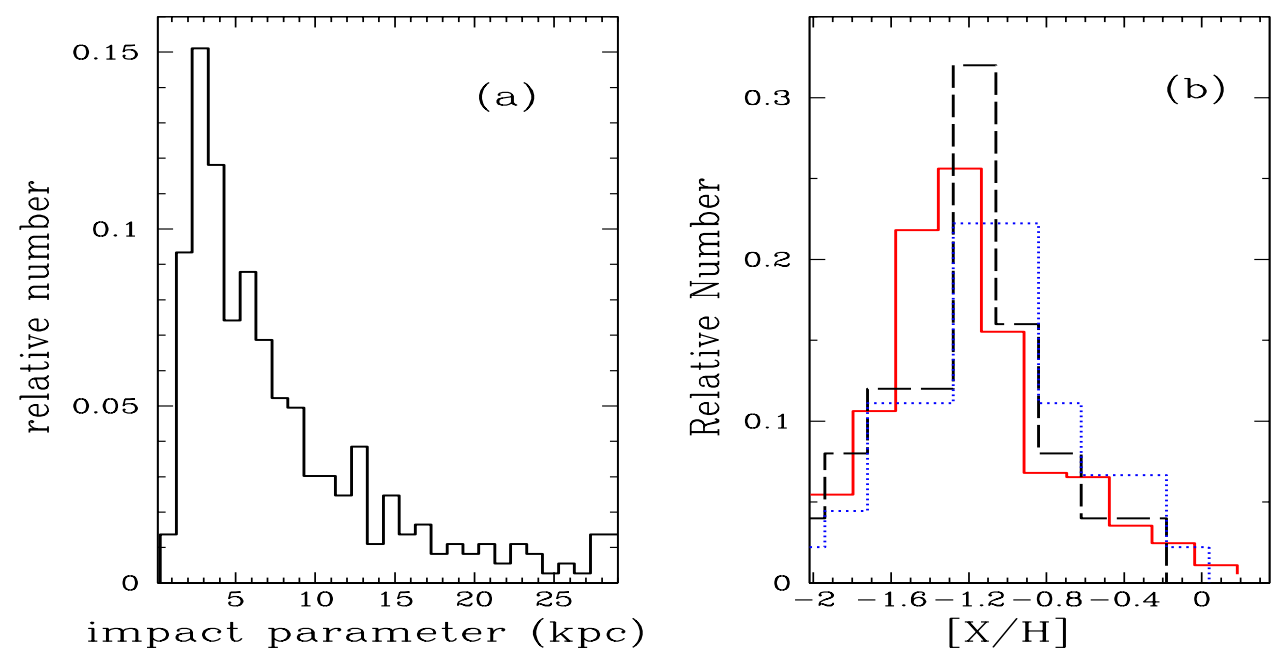

Figure 1. Distribution of impact parameters (left, a) and metallicity (right, b) of the model DLA population. In the right panel, the solid, dashed, and dotted histograms denote the model prediction, observed DLAs with $z>2$, and all DLAs respectively.

\subsection{DLA host galaxies and metallicity distribution}

In Fig. 1(a) we show the resultant distribution of the impact parameters of selected DLAs. It is found that the peak is around $3 \mathrm{kpc}$, which results from the huge amount of small halos in the PS formalism and the finite radius for individual galaxies that can produce DLAs. Because the DLAs are dominated by small galaxies which are always faint, the peak implies that the host galaxies of DLAs are difficult to observe photometrically.

The only free parameter in the present model is the effective stellar yield $p$, which is determined by comparing the predicted metallicity distribution with the observed one at a redshift greater than 2 . The model prediction for the metallicity distributions of DLAs is plotted as a solid histogram in Fig. 1(b) while the observed distributions of DLAs with $z>2$ and all DLAs are plotted as dashed and dotted histograms respectively. We get an effective yield $y=0.25 Z_{\odot}$ for the best-fit result, with the assumption of the star formation timescale for DLAs being random between 1 and 3 Gyr.

\subsection{SFR density and luminosity function of DLAs}

Based on the selected DLA sample, we can get the predicted SFR density contributed by DLAs at $z \sim 3$ and show it as a cross in the left panel of Fig. 2 which shows the cosmic SFR density as a function of redshift from a range of observations. The SFR density for DLAs with $z \gtrsim 2$ based on the CII* absorption lines are also plotted in the figure as triangles (Wolfe, Gawsier \& Prochaska 2003, hereafter WGP03). It can be found that the model prediction is consistent with observations and supports the "consensus" model described by WGP03.

In the right panel of Fig. 2, we show with the solid histogram, the predicted UV luminosity function of selected DLA hosts galaxies. The observed and dust-corrected UV luminosity functions of LBGs are also plotted as full circles (data from Adelberger \& Steidel 2000). It can be shown that the typical $R_{\mathrm{AB}}$ magnitude of the predicted DLA hosts is $\sim 30$, which is much fainter than LBGs with typical $R_{\mathrm{AB}} \sim 25$. This implies that a typical DLA host galaxy in our model has a SFR of about 100 times smaller than a typical LBG. Because the number density of DLA host galaxies is about 0.26 which 

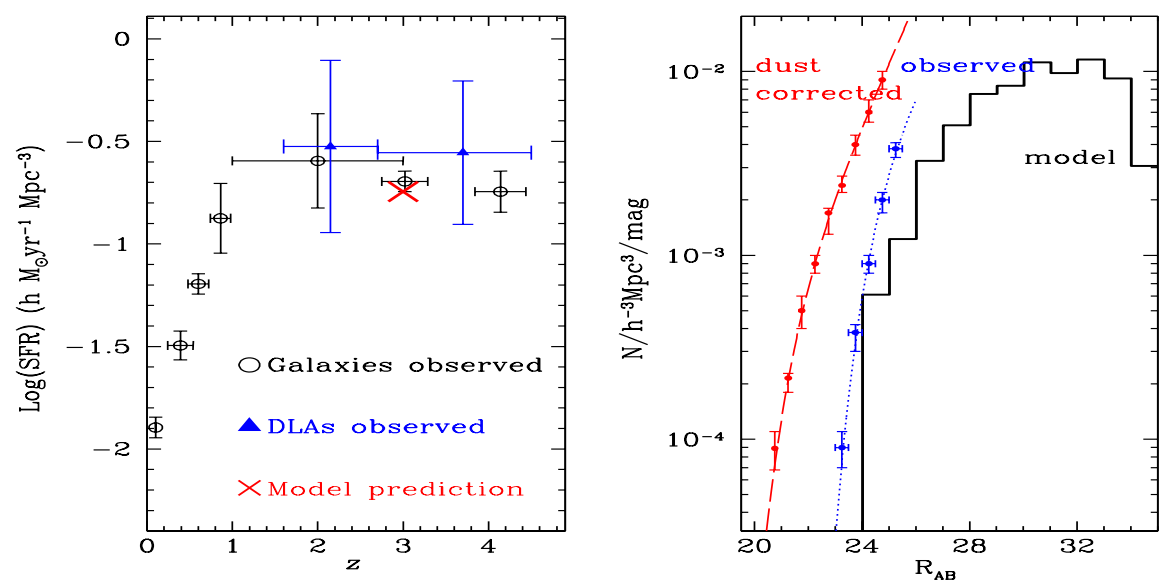

Figure 2. Cosmic SFR density as a function $z$ with the model prediction contributed by DLAs at $z \sim 3$ as a cross (left panel). The open circles and filled triangles with error bars are the observational results from galaxies and from DLAs (WGP03). The right panel shows the UV luminosity functions with the solid histogram denoting the modelled DLAs, and the dashed and dotted lines denoting LBGs with and without dust-correction which are taken from Adelberger \& Steidel (2000).

is about 100 times larger than the observed co-moving number density of LBGs, our predictions of cosmic SFR density contributed by DLAs is similar to that of LBGs at $z \sim 3$.

\subsection{HI column density distribution and relation to metallicity}

The frequency distribution of HI column density $f\left(N_{\mathrm{HI}}, z\right)$ for DLAs, which is defined as the number of absorbers per unit $N_{\mathrm{HI}}$ and per unit absorption distance $X$, is very important for understanding galaxy formation and evolution.

We plot the model prediction of $f\left(N_{\mathrm{HI}}, z\right)$ at $z \sim 3$ in the left panel of Fig. 3 as a solid line while the observed frequency distributions for DLAs with $z=2.5 \sim 3.5$ from SW00 are plotted as circles with error bars. It can be seen from the figure that the predicted distribution agrees well with observations at the high $N_{\mathrm{HI}}$ end. But it is less than the observational values at the low $N_{\mathrm{HI}}$ end, with the maximum difference as large as $3 \sigma$, i.e. the predicted distribution is a bit flatter than the observed one. A similar discrepancy appears in the more complicated numerical simulation study of Nagamine, Springel \& Hernquist (2003) as well. This could be due to the limitation of our simple model or perhaps this could be a failing of the $\Lambda$ CDM power spectrum, which deserves further investigation.

Another unusual property of DLAs is that there exists an anti-correlation between $[\mathrm{Zn} / \mathrm{H}]$ and HI column densities, which is independent of redshift as noticed by Boissé et al. (1998), who claimed that this anti-correlation is mainly due to observational selection effects due to dust. In order to test this bias, Ellison et al. (2001) surveyed a sample of DLAs toward radio selected quasars and found no significant difference in the HI distribution to those optically selected quasars. Moreover, dust obscuration has also been argued by Prochaska \& Wolfe (2002), who made a detailed analysis of dust extinction in DLAs. They found that inferred extinction values and apparent magnitudes imply that dust obscuration plays a relatively minor role in the DLA analysis at least for $z>2$. 

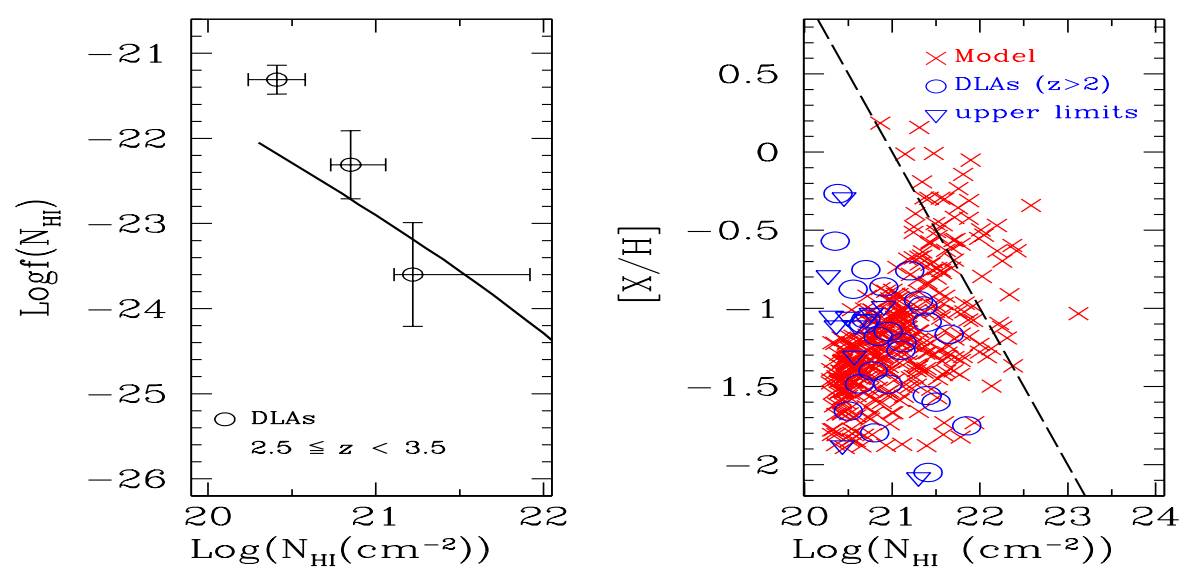

Figure 3. The left panel is $f\left(N_{\mathrm{HI}}\right)$ vs. $N_{\mathrm{HI}}$ for DLAs, with the solid line denoting the model prediction at $z \sim 3$, and the circles with error bars denoting the observations for $z=2.5 \sim 3.5$ taken from SW00. In the right panel, we show the predicted correlation between metallicity $[\mathrm{X} / \mathrm{H}]$ and $\mathrm{HI}$ column density $N_{\mathrm{HI}}$ for DLAs with crosses and open circles denoting the model DLAs and observations for $z>2$ respectively. The long dashed line is $[\mathrm{Zn} / \mathrm{H}]+\log \left(N_{\mathrm{HI}}\right)=21$.

We examined the predicted correlation between metallicity and HI column density for the selected DLA sample in Fig. 3 (right panel) with the observed results of DLAs at $z>2$ in open circles. As expected, model predictions show an opposite trend compared with observations. If we apply the proposed bias of $[\mathrm{Zn} / \mathrm{H}]+\log \left(N_{\mathrm{HI}}\right)>21$ in Fig. 3 (long dashed line), to exclude the points above this line, the difference still exists. This means that the suggestions of Boissé et al. (1998) do not help a lot in alleviating the discrepancy in our model.

Another possibility could be the inadequacy of the adopted Schmidt type star formation prescription in the model. Even for nearby galaxies, the physical basis of star formation is still poorly known. Observers have shown various empirical prescriptions for star formation in spirals (Kennicutt 1998; Wong \& Blitz 2002), and most galaxies can be fitted by a Schmidt type law. This has been widely applied in models of galaxy evolution. In fact, gas surface density $\left(\Sigma_{g}\right)$ includes both the contributions of HI $\left(\Sigma_{\mathrm{HI}}\right)$ and $\mathrm{H}_{2}\left(\Sigma_{\mathrm{H}_{2}}\right)$ with $\Sigma_{\mathrm{HI}}$ being dominant but the correlation between these two is different from galaxy to galaxy. Recent observations of star formation regions in nearby galaxies done by Wong \& Blitz (2002) showed a complex relationship between SFR and $\Sigma_{\mathrm{HI}}$. For their spiral galaxies sample (biased to molecular-rich galaxies), SFR shows virtually no correlation with $\Sigma_{\mathrm{HI}}$, suggesting a maximum HI column density around $10^{21} \mathrm{~cm}^{-2}$. This is very instructive to the star formation history of DLAs, where the observed HI column density seems have an upper limit.

\section{Summary}

We have shown in this contribution that a semi-analytical model based on the galaxy formation and evolution framework of the hierarchical structure formation scenario is quite successful in understanding DLA properties. Our simple model could reproduce well most of the observed DLA properties, such as metallicity and column density distributions, and the star formation rate density contributed by DLAs. However, we found that when the Kennicutt star formation prescription is adopted, the model always 
predicts a positive correlation between HI column density and metallicity, opposite to that observed. Different explanations exist, for example, observational bias and dust obscuration. We suggest that the observed trend of anti-correlation could most probably be physical (see also Schaye 2001). A most promising explanation might be the inadequacy of the Kennicutt star formation law in the high redshift environment. More observations and theoretical investigations are needed to clarify this trend in the future.

\section{Acknowledgements}

We are grateful to H. J. Mo and S. Boissier for their useful discussions and comments. This work was supported by NSFC10173017, 10133020, and 10073016, NKBRSF 1999075404 and NSC91-2112-M008-036.

\section{References}

Adelberger, K. L., Steidel C. C., 2000, ApJ, 544, 218

Boissé, P., Le Brun, V., Bergeron, J., Deharveng, J. M., 1998, A\&A, 333, 841

Boissier, S., Péroux, C., Pettini, M., 2003, MNRAS, 338, 131

Boissier, S., Prantzos, N., 2000, MNRAS, 312, 398

Calura, F., Matteucci, F., Vladilo, G., 2003, MNRAS, 340, 59

Cen, R., Ostriker, J. P., Prochaska, J. X., Wolfe A. M., 2003, ApJ, 598, 741

Churches, D. K., Nelson, A. H., Edmunds, M. G., 2004, MNRAS, 347, 1234

Cora, S. A., Tissera, P. B., Lambas, D. G., Mosconi, M. B., 2003, MNRAS, 343, 959

Dessauges-Zavadsky, M., Calura, F., Prochaska, J. X., D’Odorico, S., Matteucci F., 2004, A\&A, 416, 79

Ellison, S., Yan, L., Hook, I., Pettini, M., Wall, J., Shaver P., 2001, A\&A, 379, 393

Gardner, J. P., Katz, N., Weinberg, D. H., Hernquist, L., 2001, ApJ, 559, 131

Hou, J. L., Boissier, S., Prantzos, N., 2001, A\&A, 370, 23

Hou, J. L., Shu, C. G., Shen, S. Y., Chang, R. X., Chen, W. P., Fu, C. Q., 2005, ApJ, 625, in press (astro-ph/0501603)

Kennicutt, R., 1998, ApJ, 498, 541

Kulkarni, V. P., Fall, S. M., 2002, ApJ, 580, 732

Lanfranchi, G. A., Friaca, A. C. S., 2003, MNRAS, 343, 481

Mo, H. J., Mao, S. D., White, S. D. M., 1998, MNRAS, 295, 319 (MMW)

Nagamine, K., Springel, V., Hernquist, L., 2003, MNRAS, 348, 435

Okoshi, K., Nagashima, M., 2005, ApJ, 623, in press

Press, W. H., Schechter, P., 1974, ApJ, 187, 425

Prochaska, J. X., Gawiser, E., Wolfe, A. M., Castro, S., Djorgovski, S. G., 2003, ApJ, 595, L9

Prochaska, J. X., Wolfe, A. M., 2002, ApJ, 566, 68

Schaye, J., 2001, ApJ, 562, L95

Shu, C. G., Mo, H. J., Mao, S. D., 2003, astro-ph/0301035

Storrie-Lombardi, L. J., Wolfe, A. M., 2000, ApJ, 534, 552 (SW00)

Wolfe, A. M., Gawsier, E., Prochaska, J. X., 2003, ApJ, 593, 235 (WGP03)

Wong, T., Blita, L., 2002, ApJ, 569, 157 